\title{
LEIOMIOMA UTERINO ASSOCIADO A CA125 > 2000 U/ML
}

\section{UTERINE LEIOMYOMA ASSOCIATED TO CA125 > 2000 U/ML}

Diego Lopes Paim Miranda ${ }^{1}$; Carolina Matos Dórea ${ }^{1}$; Anderson Rodrigues Dourado Bastos ${ }^{1}$; Adson Roberto Santos Neves ${ }^{1,2}$; Luciana Castro Garcia Landeiro ${ }^{3}$.

\section{RESUMO}

Niveis elevados de CA125 têm sido associados a vários tipos de câncer e outras doenças benignas, tais como endometriose e leiomioma uterino. No entanto, pacientes com endometriose normalmente não apresentam niveis $>100 \mathrm{U} / \mathrm{mL}$ e um aumento significativo raramente é observado no cenário do leiomioma uterino. Uma mulher de 47 anos, nulipara, com leiomioma, apresentou-se ao nosso Departamento de Oncologia devido ao aumento de volume abdominal e elevação de CA125 (valor máximo: $2002 \mathrm{U} / \mathrm{mL}$ ). A ressonância magnética da pelve revelou miométrio heterogêneo com múltiplos nódulos (aspecto sugestivo de leiomiomatose uterina) e a tomografia por emissão de pósitrons não demonstrou aumento no metabolismo de FDG. A paciente foi submetida a histerectomia total, salpingectomia bilateral e a biópsia em cunha de ambos os ovários e o exame histopatológico confirmou o diagnóstico de endometriose e de leiomioma uterino. O período pós-operatório transcorreu sem intercorrências e o nivel sérico de CA125 voltou à normalidade após a cirurgia.

Descritores: Endometriose. Leiomioma. Biomarcadores Tumorais.

\section{ABSTRACT}

Elevated CA125 levels have been associated with various cancer types and other benign diseases, such as endometriosis and uterine leiomyoma. Even though, patients with endometriosis normally do not have levels $>100 \mathrm{U} / \mathrm{mL}$ and a significant increase is rarely seen in the scenario of uterine leiomyoma. A 47-year-old nulliparous woman, with leiomyoma, presented to our Oncology Division because of an increasing abdominal girth and an elevation of CA125 (maximum: 2002 U/mL). Magnetic resonance imaging of pelvis revealed a heterogenous myometrium with multiple nodules and positron emission tomography/computed tomography demonstrated no increased FDG metabolism. The patient was submitted to total hysterectomy, bilateral salpingectomy and a wedge biopsy of both ovaries and histopathological examination confirmed the endometriosis and uterine leiomyoma. Her postoperative period was uneventful and serum CA125 level came to normal after surgery.

Keywords: Endometriosis. Leiomyoma. Biomarkers, Tumor.

\section{INTRODUÇÃO}

O biomarcador tumoral CA125 é uma glicoproteína de alto peso molecular, originada no epitélio celômico ${ }^{1}$. Niveis elevados de CA125 estão associados a diversos tipos de câncer, tais como câncer de mama, de pulmão e, mais frequentemente, câncer de ovário ${ }^{2}$. Por outro lado, níveis aumentados também têm sido associados a outras doenças benignas, como endometriose e leiomioma ${ }^{3-6}$.

$\mathrm{Na}$ prática clínica, encontra-se intimamente associado ao câncer de ovário, uma vez que até $80 \%$ dos casos desse tipo de câncer cursam com niveis elevados de $\mathrm{CA} 125^{2}$. Conforme recomendações da Socie- dade Europeia de Oncologia (ESMO), a dosagem de CA125 de rotina é recomendada para diagnóstico do carcinoma epitelial de ovário (CEO) e para avaliação clínica de sua recorrência ${ }^{2}$.

Quanto ao uso deste biomarcador no rastreio do câncer de ovário, estudos falharam em demostrar redução de mortalidade quando realizado na população geral7. No que diz respeito a idade, foi encontrada maior sensibilidade do CA125 para CEO em mulheres na pré-menopausa e maior especificidade em pacientes na pós-menopausa, visto que outras patologias no período prémenopausa também são responsáveis por alterações no CA1258,9.

\footnotetext{
${ }^{1}$ Universidade Federal da Bahia - Faculdade de Medicina - Salvador, BA, Brasil.

${ }^{2}$ Hospital da Mulher - Salvador, Bahia, Brasil.

${ }^{3}$ Grupo Oncoclínicas - Núcleo de Oncologia da Bahia - Salvador, BA, Brasil.
} 
Endometriose é uma condição benigna caracterizada pela presença de glândulas endometriais funcionais e seu estroma fora da cavidade uterina ${ }^{10}$. Atualmente, estima-se que esta patologia está presente em aproximadamente $10-15 \%$ das mulheres na pré-menopausa e a literatura sugere que níveis séricos de CA125 são consideravelmente maiores em mulheres que apresentam doença moderada ou grave ${ }^{10,11}$. Contudo, na literatura, niveis séricos de CA125 > $1000 \mathrm{U} / \mathrm{mL}$ foram relatados de forma pontual em pacientes com endometriose ${ }^{11}$.

Pacientes com leiomioma uterino também podem apresentar elevação dos níveis séricos de CA1256,12. Entretanto, também são raros os relatos de CA125 > $1000 \mathrm{U} / \mathrm{mL}$ em associação a leiomiomas uterinos $^{6,11}$. Leiomiomas uterinos são tumores ginecológicos benignos comuns, que afetam cerca de $60-80 \%$ das mulheres na pré-menopausa, sendo queixa frequente em consultas ginecológicas ${ }^{4,12}$. Aproximadamente $20 \%$ dos leiomiomas são sintomáticos e necessitam de tratamento, no entanto, marcadores tumorais não são utilizados para diferenciação ou monitoramento dessa doença ${ }^{12}$.

A etiologia precisa do leiomioma não é bem estabelecida. O estrógeno é capaz de estimular seu crescimento, podendo ser evidenciado após a menarca, progredindo durante a gestação e reduzindo após a menopausa, com recrescimento após o início de terapia hormonal ${ }^{4}$. Diante disso, niveis elevados de CA125 foram associados com, ao menos, um mioma com diâmetro $\geq 5 \mathrm{~cm}$, além de adenomiose associada ${ }^{12}$.

Este relato apresenta o caso de uma paciente de 47 anos com leiomioma uterino e endometriose associados a niveis extremamente elevados de CA125 (valor máximo: $2002 \mathrm{U} / \mathrm{mL}$ ).

\section{RELATO DO CASO}

Uma mulher nulípara de 47 anos de idade, com história de leiomioma uterino, apresentou-se ao nosso Departamento de Oncologia com queixa de aumento progressivo da circunferência abdominal e elevação de CA125.

No momento da consulta, referiu não estar tomando nenhum medicamento, mas relatou histórico de uso de pílulas contraceptivas com baixa dosagem hormonal entre os 18 e 20 anos, e histórico familiar de câncer de mama (duas tias paternas com < 60 anos aos diagnóstico). Negou antecedentes médicos e uso de tabaco, álcool ou outras drogas. Ela foi submetida a painel de genes de alto risco para câncer de mama e ovário hereditários, o qual resultou negativo para mutações patogênicas (37 genes avaliados por NGS).

Os marcadores tumorais obtidos na época demonstraram um nivel sérico máximo de CA125 de $2002 \mathrm{U} / \mathrm{mL}$, que aumentou significativamente desde a primeira medida (129 U/mL), 5 anos antes. (Tabela $1)$.

$\mathrm{Na}$ ressonância magnética da pelve (abril/2019) observou-se útero aumentado e miométrio heterogêneo com contorno lobulado e múltiplos nódulos (maior: $8,3 \times 7,2 \mathrm{~cm})$. A tomografia por emissão de pósitrons (PET-CT) com o uso do traçador fluordeoxiglicose (FDG) em junho/2019 mostrou útero aumentado, com imagem nodular hipoatenuante na região anexial direita, de $3,2 \times 1,8 \mathrm{~cm}$, associada a um foco hipermetabólico em sua periferia (SUV: 4.3) - possivelmente representando alterações fisiológicas - e outra imagem hipoatenuante nodular inferior à mencionada acima, medindo $2,8 \times 1,9 \mathrm{~cm}$, sem aumento do metabolismo de FDG.

Em agosto/2019, foi submetida a histerectomia total, salpingectomia bilateral e biópsia em cunha de ambos os ovários. $\mathrm{O}$ exame histopatológico confirmou a presença de pequenos focos de endometriose e leiomiomas uterinos. O período pósoperatório transcorreu sem intercorrências e o valor sérico de CA125 normalizou após a cirurgia. No seguimento, após três meses da cirurgia, os níveis de CA125 ainda estavam normais $(12 \mathrm{U} / \mathrm{mL})$.

\section{DISCUSSÃO}

O CA125 foi identificado como antígeno do câncer de ovário em 1981 e, posteriormente, desenvolvido como biomarcador para detecção precoce do CEO. Através de imunohistoquímica, mostrou-se estar localizado no epitélio das tubas uterinas, endométrio, cérvice e células mesoteliais da pleura, pericárdio e peritônio ${ }^{12,13}$. Niveis séricos > $35 \mathrm{U} / \mathrm{mL}$ foram encontradas em mais de $80 \%$ de pacientes com CEO e em apenas $1 \%$ de mulheres saudáveis ${ }^{9}$. Uma dosagem acima de $200 \mathrm{U} / \mathrm{mL}$ foi definida como critério positivo para diferenciação entre massas pélvicas benignas e malignas 
em mulheres na pré-menopausa, com menos de 50 anos de idade ${ }^{13}$.

Apesar de niveis mais altos de CA125 estarem associados a CEO, niveis aumentados foram também atribuídos a diversas patologias benignas ginecológicas e nãoginecólogicas, tais como endometriose, leiomioma, doença inflamatória pélvica, cirrose hepática e outras neoplasias malignas não-ginecológicas $4,5,13$. $\mathrm{O}$ valor preditivo positivo do CA125 (> $95 \mathrm{U} / \mathrm{mL}$ ) para câncer de ovário é de $96 \%$ entre mulheres na pósmenopausa, com altas sensibilidade e especificidade ${ }^{9}$. Apesar disso, este biomarcador tem baixa especificidade entre mulheres na pré-menopausa.

Endometriose é uma das doenças benignas mais comumente associadas ao aumento do nivel sérico de CA125, no entanto pacientes normalmente não apresentam valores $>100 \mathrm{U} / \mathrm{mL}^{8,11}$. Niveis séricos refletem progressão e gravidade do quadro $^{14}$. Sabe-se, ainda, que há queda e normalização após tratamento cirúrgico ou clínico adequado ${ }^{2}$. No entanto, dosagem de CA125 de rotina não é recomendada para diagnóstico ou tratamento de endometriose ${ }^{15}$.

O leiomioma uterino é a neoplasia ginecológica mais comum em mulheres na idade reprodutiva ${ }^{13}$. Existem poucos relatos na literatura de associação entre altos níveis de CA125 e leiomioma uterino $4,5,10,13$. Diante disso, uma elevação tal como a descrita neste relato de caso é raramente observada no cenário do leiomioma uterino. Frequentemente, há queda acentuada e normalização dos níveis de CA125 após miomectomia ${ }^{13}$.

Neste relato de caso, é descrito aumento expressivo e progressivo de CA125 (maior nivel: $2002 \mathrm{U} / \mathrm{mL}$ ) em uma paciente com leiomioma e pequenos focos de endometriose. Não foram evidenciadas alterações histopatológicas associadas a malignidade e os niveis de CA125 normalizaram após procedimento cirúrgico.

Tal como ilustrado neste relato de caso, niveis de CA125 significativamente aumentados podem se associar a patologias benignas ginecológicas e não-ginecológicas, como endometriose e leiomioma4,5,13. No entanto, existem poucos casos na literatura de associação entre leiomioma e níveis séricos tão elevados de CA125 (2002 U/mL). Neste caso, torna-se evidente que no diagnóstico diferencial de uma massa de tama- nho acentuado (atentar para as características radiológicas) com niveis extremamente altos de CA125 em uma mulher na idade reprodutiva, deve-se considerar também patologias benignas como leiomiomas e/ou endometriose, apesar da raridade da associação entre níveis tão elevados de CA125 com patologias benignas, como no caso aqui descrito.

\section{REFERÊNCIAS}

1. Rao S, Kapurubandara S, Anpalagan A. Elevated CA 125 in a case of Leaking Endometrioma. Case Rep Obstet Gynecol. Published online: 12 Sep 2018; DOI: $10.1155 / 2018 / 2385048$.

2. Ledermann JA, Raja FA, Fotopoulou C, Gonzalez-Martin A, Colombo N, Sessa C. Newly diagnosed and relapsed epithelial ovarian carcinoma: ESMO Clinical Practice Guidelines for diagnosis, treatment and followup. Annals of Oncology 2013, Oxford: Oxford University Press: 10.1093.

3. R. L. Barbieri, J. M. Niloff, R. C. Bast Jr., E. Scaetzl, R. W. Kistner, and R. C. Knapp. Elevated serum concentrations of CA-125 in patients with advanced endometriosis. Fertil Steril. 1986 May;45: 630-634.

4. Jao MS, Huang KG, Jung SM, Hwang LL. Postmenopausal uterine leiomyoma with hemorrhagic cystic degeneration mimicking ovarian malignancy. Taiwan J Obstet Gynecol. 2007 Dec;46:431-434.

5. Ghamande SA, Eleonu B, Hamid AM. High levels of CA-125 in a case of a parasitic leiomyoma presenting as an abdominal mass. Gynecol Oncol. 1996 May;61:297-298.

6. Dunn JS Jr, Anderson CD, Method MW, Brost BC. Hydropic degenerating leiomyoma presenting as pseudoMeigs syndrome with elevated CA 125. Obstet Gynecol; 1998 Oct;92:648-649.

7. Kamal R, Hamed S, Mansour S, Mounir Y, Abdel-Sallam S. Ovarian cancer screening-ultrasound; impact on ovarian cancer mortality. $\mathrm{Br} \mathrm{J}$ Radiol. Published online: 04 Sep 2018; DOI: 10.1259/bjr.20170571

8. Terlikowska KM, Dobrzycka B, Witkowska AM, et al. Preoperative HE4, CA125 and ROMA in the differ- 
ential diagnosis of benign and malignant adnexal masses. J Ovarian Res. Published online:19 Jul 2016. DOI:10.1 186/s13048-016-0254-7.

9. Bast RC Jr, Feeney M, Lazarus $\mathrm{H}$, Nadler LM, Colvin RB, Knapp RC. Reactivity of a monoclonal antibody with human ovarian carcinoma. J Clin Invest. 1981 Nov:68(5):13311337.

10. Agic A. Combination of CCR 1 mRNA, MCP1, and CA125 measurements in peripheral blood as a diagnostic test for endometriosis. Reprod Sci. 2008 Nov;15:906-911.

11. Shiau CS, Chang MY, Chiang $\mathrm{CH}$, Hsieh CC, Hsieh TT. Ovarian endometrioma associated with very high serum CA-125 levels. Chang Gung Med J. 2003 Sep;26(9):695-699.

12. Babacan A, Kizilaslan C, Gun I, Muhcu M, Mungen E, Atay V. CA 125 and other tumor markers in uterine leiomyomas and their association with lesion characteristics. Int $\mathrm{J}$ Clin Exp Med. 2014 Apr;15:1078-1083.
13. Rani AK, Kapoor D. Ruptured ovarian endometrioma with an extreme rise in serum CA 125 level - A case report: Ovarian endometrioma with very high CA-125 level. Gynecol Oncol Case Rep. Published online 19 May 2012; DOI: 10.1016/j.gynor.2012.05.005.

14. Kahraman K, Ozguven I, Gungor M,Atabekoglu CS. Extremely elevated serum CA-125 level as a result of unruptured unilateral endometrioma: the highest value reported. Fertil Steril. 2007 Oct;88(4): 968.e15968e 17.

15. Jacobsand I. Bast RC Jr. The CA 125 tumour-associated antigen: a review of the literature. Hum Reprod. 1989 Jan;4: 1-12.

Recebido em: 27/05/2020

Aceito para publicação: 04/06/2020

Conflito de interesses: Não

Endereço para correspondência:

Diego Lopes Paim Miranda

E-mail: diegolpmiranda@hotmail.com 